\title{
Vietnamese American Women's Beliefs and Perceptions on Cervical Cancer, Cervical Cancer Screening, and Cancer Prevention Vaccines: A Community-Based Participatory Study
}

Asian/Pacific Island Nursing Journal Volume 2(4): 133-142

(C)Author(s) 2017

http://digitalscholarship.unlv.edu/apin/

\author{
Connie Kim Yen Nguyen-Truong, PhD, RN, PCCNa; Kim Quy Vo Nguyen, DNP, FNP-C \\ Thai Hien Nguyen, BSN, RN'; Tuong Vy Le, MS $^{b}$; Anthony My Truong, BS, RPh ${ }^{b}$; Keara \\ Rodela, MPH, CHW'; and Rachael Allan, BSN, RN ${ }^{\mathrm{a}}$
}

\begin{abstract}
Cervical cancer remains commonly diagnosed in Vietnamese American women. Despite efforts to increase cervical cancer screening among Vietnamese American women, participation rates are persistently lower than the national goal. The objective of this study is to explore beliefs of Vietnamese American women about cervical cancer, cervical cancer screening, and cancer prevention vaccines. A qualitative descriptive investigation captured group perceptions about meaning and beliefs of cervical cancer, screening, and cancer prevention vaccines, and participants' stories using a community-based participatory research approach.

Forty Vietnamese American women were recruited from the Portland, Oregon metropolitan area into four focus groups. Using a process of directed content analysis, focus group transcripts were coded for themes. We found that cervical cancer continues to be a difficult topic to discuss, and Vietnamese American women may not bring the topic up themselves to their health care providers. Some women experienced intense emotions of fear or shame of having their cervix examined. Women delayed seeking cervical cancer screening and needed to have early warning signs, which guided them as to when to seek health care. Women focused on cleanliness through vaginal and/or perineal washing as primary prevention for cervical cancer. There were limited awareness and knowledge about cancer prevention vaccines, specifically the human papillomavirus. Some women relied heavily on their informal social networks of family, friends, or community for health knowledge.

Fear and misunderstanding dominated the beliefs of Vietnamese American women about cervical cancer screening and prevention. These findings underscored the importance of having culturally-specific findings, which will inform a multicomponent intervention to promote cervical cancer screening and cancer prevention vaccine uptake within this population.
\end{abstract}

Keywords: Family practice nursing, public health and community nursing, community health and preventive medicine, women's health, Vietnamese, cervical cancer, screening, vaccine, beliefs, qualitative, focus groups, community-based participatory research

Vietnamese American (VA) women are twice as likely $(16.8 / 100,000)$ to be diagnosed with cervical cancer (CC) than White non-Hispanic women (8.1/100,000; Miller, Chu, Hankey, \& Ries, 2008). The best method for early detection of CC is to have regular screening with a Papanicolaou (Pap) test, which may include a test for human papillomavirus (HPV; American Cancer Society, 2015). Early CC detection greatly improves treatment outcomes with survival rates at nearly $100 \%$ for precancerous lesions and $91 \%$ for those diagnosed with localized CC five-years post-diagnosis (American Cancer Society,

\author{
${ }^{a}$ College of Nursing, Washington State University, Vancouver, \\ WA, USA \\ ${ }^{b}$ Vietnamese American Community, Portland, OR, USA \\ ${ }^{c}$ Immigrant \& Refugee Community Organization Asian Family \\ Center, Portland, OR, USA \\ ${ }^{d}$ Immigrant \& Refugee Community Organization, Portland, OR, \\ USA \\ Corresponding Author: \\ Dr. Connie Kim Yen Nguyen-Truong, PhD, RN, PCCN \\ College of Nursing, Washington State University, Vancouver, WA \\ 14204 NE Salmon Creek Avenue, Vancouver, WA, 98686-9600 \\ Email: c.nguyen-truong@wsu.edu
}


2015). Despite the importance of early CC detection, screening rates are persistently low among VA women. In prior studies, $69-81 \%$ of VA women reported being up-to-date with CC screening (Nguyen-Truong et al., 2012; Thompson et al., 2014), although the Healthy People 2020 CC screening goal is $93 \%$ of all women aged 21 to 65 years (HealthyPeople.gov, 2017). The Centers for Disease Control and Prevention (2016) recommend cancer prevention vaccines as an effective prevention strategy, including CC (HPV).

Cancer vaccination disparities exist for Asian American women as compared to the general population (Lee et al., 2015). Particularly in VA immigrant women, $14 \%$ and $10 \%$ reported initiation of HPV vaccination and series completion, respectively (Yi, Anderson, Le, Escobar-Chaves, \& Reyes-Gibby, 2013). Researchers found that VA immigrant women with limited English literacy skills, lower educational levels, and financial barriers were less likely to have cancer vaccine acceptance and believe in being susceptible to developing CC (Strong, Lee, Tanaka, \& Juon, 2012; Yi et al., 2013). VA immigrant women who reported respect for their health care provider (HCP)s' opinions were more likely to accept medical recommendations for cancer prevention vaccinations (Strong et al., 2012; Yi et al., 2013).

Although the literature tends to aggregate Asian Americans, Asian Americans are a heterogeneous population with origins from over 50 different countries and more than 100 languages (Gomez et al., 2013). Research supports the need to study subgroups of Asian Americans. VA women have unique cultural health beliefs and practices than other Asian American subgroups, which may influence their cancer risks and outcomes (Solanki, Ko, Qato, \& Calip, 2016). In Chawla, Breen, Liu, Lee, and KagawaSinger's (2015) study of Asian American women, they found that non-English speaking VA women or women with limited English proficiency were less likely to pursue regular $\mathrm{CC}$ screening than Filipino, Japanese, Chinese, and Korean women. Also, VA women who reported an income less than $200 \%$ below the federal poverty level were less likely to obtain regular $\mathrm{CC}$ screening than other Asian American women (Chawla et al., 2015). Our previous qualitative study reported that VA women relied on personal health regimens such as vaginal washing as a means of CC prevention (Gregg, Nguyen-Truong, Wang, \& Kobus, 2011). Hence, VA women may need culturally and linguistically appropriate counseling.

Overall, we must address VA women's culturally specific beliefs and practices in the design of appropriate interventions. Thus, the purpose of the community-based participatory research (CBPR) qualitative descriptive investigation was to explore beliefs of VA women about CC, CC screening, and cancer prevention vaccines.

\section{Theoretical Framework}

We used an investigator-modified, openended, semi-structured question guide, and incorporated topics guided by the Health Belief Model (HBM; Lee-Lin, Menon, Nail, \& Lutz, 2012). The HBM framework emphasizes individual health beliefs and health behavior change (Champion \& Skinner, 2008). The HBM has been used in studies of Asians and Asian Americans (Ho et al., 2005; LeeLin et al., 2012; Lee-Lin, Nguyen, Pedhiwala, Dieckmann, \& Menon, 2015).

The question guide included the following HBM topics (concepts), which appeared to influence CC screening beliefs and practices in the current study: perceived susceptibility, perceived benefits, and perceived barriers (Champion \& Skinner, 2008). Perceived susceptibility was defined as the "belief about the chances of experiencing a risk or getting a condition or disease" (Champion \& Skinner, 2008, p. 48). For example, a VA woman is more likely to participate in $\mathrm{CC}$ screening if she believes she is susceptible to developing $\mathrm{CC}$ or is more likely to vaccinate if she believes she is susceptible to acquiring a HPV infection. She is also more likely to vaccinate her children if she believes the children will be susceptible to developing HPV-associated cancer or acquiring a HPV infection. Perceived benefits were defined as the "belief in efficacy of the advised action to reduce risk or seriousness of impact" (Champion \& Skinner, 2008, p. 48). Perceived barriers were defined as the "belief about the tangible and psychological costs of the advised action" (Champion \& Skinner, 2008, p. 48). For example, a VA woman is more likely to participate in CC screening if she sees benefits with $\mathrm{CC}$ screening and few barriers, or is more likely to vaccinate if she sees benefits and few barriers in HPV vaccination. She is also more likely to vaccinate her children if she sees benefits and few barriers. The HBM also suggests that having adequate knowledge is important (Champion \& Skinner, 2008). A limitation to the HBM is its sole emphasis on cognition without attending to the influence of emotions on screening behavior (Champion \& Skinner, 2008).

\section{Method}

\section{Research Ethics}

The local Washington State University Institutional Review Board (3005) determined the study as exempt. A study information sheet that included the study's purpose and procedures did not require participants to sign their names to demonstrate consent for study enrollment. As a part of the informed 
consent process, a VA bicultural and bilingual health educator/research assistant (third author) provided the study information sheet and explained the study's purpose and procedures for participation. The third author also explained the lack of any known risks or discomforts, personal benefits associated with participation, and that participation was voluntary and confidential. The participants demonstrated having consented to take part in the study by completing the sociodemographic form and attending a focus group.

\section{Design}

A CBPR design was used to conduct the qualitative descriptive study using focus groups, which involved a collaboration between community and academic partners to address an identified public health priority issue, CC screening and prevention (Israel, Eng, Schulz, \& Parker, 2012). The Immigrant \& Refugee Community Organization (IRCO; 2017) and their Asian Family Center, a community-based organization, and the principal investigator (PI, first author) have had a CBPR partnership since 2008. The research team used a list of community agreements (e.g., it is okay to agree or not be in agreement), which was based on mutual trust and on understanding how the research team would work and learn collaboratively (Nguyen-Truong, Tang, \& Hsiao, 2017).

The CBPR design included empowerment of community member partners to be involved in conducting research to build research skills. Together, the community member partners and the PI (a) defined the current study's purpose; (b) adapted and modified the open-ended, semi-structured question guide for use with focus groups; (c) translated study materials; (d) recruited or referred participants; (e) co-conducted focus groups; (f) transcribed and translated data; (g) analyzed data; (h) disseminated the findings to the VA community; and (i) prepared this article for the scientific community. Community partners' contributions added cultural and linguistic appropriateness to the research rigor.

\section{Sample}

We recruited VA women from a local community-based organization and from the VA community in the Portland, Oregon metropolitan area from October 2016 to April 2017. Purposive and snowball sampling were used to recruit the participants. Referrals were requested from our community participatory advisory council team and recruited participants at IRCO and their Asian Family Center, as well as the VA community. IRCO (2001-2017) has 40 years of history and experience working with immigrants and refugees and communities-at-large in Oregon. We created a study advertisement flyer printed in Viet- namese and English and team members conducted outreach. Outreach included staff working with VA women within IRCO and its reception waiting areas. The flyers were also posted/distributed at Asian grocery stores, HCPs' offices, and several community outreach events (e.g., Vietnamese New Year). Snowball sampling relied on direct referrals from the participants.

The qualitative descriptive sample size of 40 was determined based on the study's purpose, the scope of the topics, sampling, and the quality of data obtained from the focus groups (Hennink, Kaiser, \& Marconi, 2017). We screened 46 VA women for eligibility, one of which was not eligible, she had undergone a hysterectomy with removal of the cervix. Of the $45 \mathrm{VA}$ women enrolled in the study, five later decided that they no longer wanted to participate due to a schedule conflict. Participants included those who self-identified as Vietnamese or VA, immigrated to the United States or were U.S.-born, with no personal history of CC or breast cancer, not having had a hysterectomy with removal of the cervix, and being able to understand or read Vietnamese or English.

\section{Procedures}

The study materials (advertisement flyer, consent form, sociodemographic form, open-ended and semi-structured question guide) were translated into Vietnamese using a team translation approach. The third author translated the study materials into Vietnamese. Next, the PI, the assistant project coordinator (fourth author; graduate degree in counseling and background experience as a Vietnamese language teacher at a faith-based organization), and a VA community consultant (graduate degree in public and health administration) reviewed the translated study materials and identified grammar, clarity, and logical flow issues. Next, the team had discussions, resolved ambiguities, and arrived at a consensus for a meaningful translation. The team process improved the quality of translation above a literal translation, which was similar to our prior study (Nguyen-Truong et al., 2015).

The participants completed consent and sociodemographic forms, available in both Vietnamese and English. The current authors used focus groups as the data collection method. A focus group method was a good fit to the current study because it captured group perceptions about meaning and beliefs of $\mathrm{CC}$, $\mathrm{CC}$ screening, cancer prevention vaccines, and participants' stories (Morgan \& Bottorff, 2010). Each participant attended one focus group. Four focus groups were conducted consisting of seven to 15 participants per group. Participants could choose to attend a focus group conducted in Vietnamese or English, and all chose Vietnamese. Thus, we conducted all focus groups in Vietnamese. The focus groups lasted about 90-120 minutes. 
Asian/Pacific Island Nursing Journal, Vol. 2, Iss. 4 [2017], Art. 2

Table 1. Sociodemographic and Background Characteristics of Participants $(\mathrm{N}=40)$

\begin{tabular}{|c|c|c|}
\hline Characteristic & & Mean $(S D)$ \\
\hline Age, years & & $46.45(12.93)$ \\
\hline Age at immigration, years & & $35.18(14.36)$ \\
\hline Years lived in the United States of America & & $11.65(10.98)$ \\
\hline Characteristic & $n$ & $(\%)$ \\
\hline \multicolumn{3}{|l|}{ Birth Place } \\
\hline Central Vietnam & 4 & $(10.0)$ \\
\hline South Vietnam & 35 & $(87.5)$ \\
\hline United States of America & 1 & $(2.5)$ \\
\hline \multicolumn{3}{|l|}{ Marital Status } \\
\hline Married & 32 & $(80)$ \\
\hline Single and Never married & 2 & $(5.0)$ \\
\hline Separated & 3 & $(7.5)$ \\
\hline Divorced & 1 & $(2.5)$ \\
\hline Widowed & 2 & $(5.0)$ \\
\hline \multicolumn{3}{|l|}{ Education } \\
\hline Elementary & 2 & $(5.0)$ \\
\hline Some middle school & 6 & $(15.0)$ \\
\hline Some high school & 13 & $(32.5)$ \\
\hline Some college & 6 & $(15.0)$ \\
\hline Graduated from college & 13 & $(32.5)$ \\
\hline \multicolumn{3}{|l|}{ Employment } \\
\hline Full-time & 13 & $(32.5)$ \\
\hline Part-time & 9 & $(22.5)$ \\
\hline Not employed & 18 & $(45.0)$ \\
\hline \multicolumn{3}{|l|}{ Household income before taxes } \\
\hline$<\$ 15,000$ & 10 & $(25.0)$ \\
\hline$\$ 15,000-30,000$ & 12 & $(30.0)$ \\
\hline$\$ 30,001-50,000$ & 10 & $(25.0)$ \\
\hline$\$ 50,001-75,000$ & 4 & $(10.0)$ \\
\hline$\$ 75,001-100,000$ & 2 & $(5.0)$ \\
\hline Not Sure & 5 & $(12.5)$ \\
\hline \multicolumn{3}{|l|}{ How well do you speak English? } \\
\hline Not at all & 6 & $(15.0)$ \\
\hline Poorly & 9 & $(22.5)$ \\
\hline Average & 14 & $(35.0)$ \\
\hline Well & 6 & $(15.0)$ \\
\hline Fluently like a Native English Speaker & 12 & $(12.5)$ \\
\hline \multicolumn{3}{|l|}{ Do you have any kind of health care coverage? } \\
\hline No & 1 & $(2.5)$ \\
\hline Yes $^{\mathrm{a}}$ & 39 & $(97.5)$ \\
\hline
\end{tabular}

Note. $S D=$ standard deviation. Of the 39 participants who responded yes, $n=3(7.6 \%)$ participants have health insurance coverage for emergency room visits only.

The focus groups were conducted at a convenient location (IRCO's Asian Family Center location) and near public transportation. None of the participants had previously participated in a focus group. Before conducting the focus groups, the PI led a dinámica, which is a social learning game that helped participants to feel at ease (Nguyen-Truong et al., 2017; Wiggins, 2011). The PI invited participants to stand and engaged them in a song with choreographed movements (e.g., clapping hands). The PI used the following song verse, which was previously done with the community partners, "We [single movement] our [body part] together. We [single movement] our [body part] together. Hey - Hey We [single movement] our [body part] together..." (Nguyen-Truong et al., 2017, p. 218).

An open-ended, semi-structured question guide was used to conduct the focus groups and included questions about knowledge and beliefs about $\mathrm{CC}, \mathrm{CC}$ screening, and cancer prevention vaccines. The focus groups were audio recorded. Each focus group was co-conducted by the PI and another trained facilitator (third author). The PI had extensive experience in clinical nursing, conducting focus groups, training, community leadership, community assessments, and translating with VAs. The other focus group facilitator had experience in clinical nursing and was trained to co-conduct focus groups. 
Although there were various sizes across the focus groups, the PI and the other trained focus group facilitators were able to facilitate the discussion so that all participants had an opportunity to share within their respective focus group. We provided food during focus groups as a form of hospitality. At the end of the focus group, each participant received a \$20 grocery gift card as a thank you.

\section{Data Analysis}

The focus groups were designed to investigate the beliefs and perceptions of VA women on $\mathrm{CC}, \mathrm{CC}$ screening, and cancer prevention vaccines. We used a theory-directed approach to content analysis, which is more of a deductive, structured approach than a conventional content analysis (Hsieh \& Shannon, 2005). Thus, we analyzed focus group transcripts guided by the HBM as described earlier.

We used a team approach throughout the data analysis. One team member transcribed each focus group verbatim in Vietnamese and then reviewed each focus group a second time to verify the accuracy of the transcription. Then, the PI reviewed the transcriptions for accuracy. Next, other team members translated the transcripts into English. The team members then compared the English and Vietnamese versions. Finally, we held team meetings to review the translations, discuss and resolve ambiguities, and obtain a consensus regarding narrative meanings.

The data were managed using ATLAS.ti (version 7.5.18) qualitative software. A coding scheme was developed based on the open-ended, semi-structured question guide. Before the current study, an independent consultant with a background in qualitative data analysis and research with Asian Americans reviewed the definitions. This ensured the accuracy of predetermined categories that included the focus areas of the HBM described earlier (Hsieh \& Shannon, 2005). Two team members, both of whom are VA bicultural and bilingual, independently coded the first translated English focus group transcript. Next, the team met to discuss discrepancies and agreed on the best codes to improve consistency. Three team members independently coded the other English focus group transcripts. After that, the coding was reviewed by and discussed with the PI, and they agreed upon the best coding. Next, we compared the codes for each focus group across the transcripts and identified the main themes.

The team discussed the data analysis with the VA community advisors, who had backgrounds in medicine, public and health administration, and biology, as well as with the VA women community members. In that capacity, they served as external auditors. Trustworthiness of the analysis was assured by checking the English translations against the Vietnamese transcriptions. We ensured credibility through the research team's review of the transcripts, and through peer debriefing (Lincoln \& Guba, 1985). The team approach to data analysis allowed for indepth discussions about shared and different understandings about the data. This ensured rigor in the data analysis, as the research team considered cultural and language insights throughout their review.

\section{Results}

Table 1 displays the sociodemographic and background characteristics of the participants. Five main themes were identified from the participants: 1) fear of CC and CC screening; 2) "abnormal bleeding or having abnormal discharge, I must have an examination"; 3) vaginal and/or perineal washing to prevent cervical cancer or infection; 4) limited cancer prevention vaccine awareness and knowledge; and 5) decision-making influences about cancer prevention vaccines.

\section{Main Themes}

\section{Fear of CC and CC Screening}

Fear of talking about CC.

Some participants discussed having a fear of talking about CC and cancer in general. For example:

People are shy and afraid of talking about cervical cancer. They don't understand what cancer is ... I know two people went to remove the cervix, but they removed ... fibroid tumor ... They are not afraid talking about that. My close friends talked about that when I asked them. As for cancer, people keep silent and don't talk about it.

Private Areas - Fear or Shame of "Being Seen."

Some participants discussed having fear or feeling shame if they were to have their private areas seen, even if it was a woman's health exam with either a male or female HCP. This was a primary barrier to having regular $\mathrm{CC}$ screening. The following description shares one woman's feeling of shame where multiple HCPs versus just one performed her $\mathrm{CC}$ screening:

In Vietnam, I had an exam that is very awkward, very crowded, interns in there while doctor processed a woman's health exam. Those male interns kept standing there made me being shameful ... They came to practice making me feeling shameful so that I wanted to die. Lying and spreading legs widely, then those interns stayed in there ... but not in the United States ... they cover me properly, there is only doctor and me...

Although one woman was afraid and shy to have an exam on her private areas, she decided to do so be- 
cause of a friend who had died. She shared her concerns about having a $\mathrm{CC}$ screening:

[Afraid] of being seen - my private areas [including cervix] .... I was so scared when I was young, but later on I learned oh, if not [have CC screening] having cancer, [and] adding to the death of my friend ... so I must have an exam .... I am afraid of having an exam [CC screening], afraid of having an exam on private areas, very afraid! I am shy ... I think that secretive habits of Vietnamese women make them shy.

\section{"Abnormal Bleeding or Having Abnormal Discharge, I Must Have an Examination"}

Some participants talked about having abnormal bleeding or white blood/discharge as a sign of $\mathrm{CC}$, cancer, disease, or infection and the need to get it checked by a HCP. "If I feel abnormal bleeding or having abnormal discharge, I must have an examination because it is a sign of cancer." Another described:

When I was in Vietnam, I have a cousin who went to have a general examination to ask ... [about the] white discharge coming out from her vagina in clots .... Doctor said she had cancer stage one .... The first follow up is after five years. Doctor said that she's already had two children so she should cut it [surgery] ... she ... cut.

Another woman shared what white blood is:

White blood ... is mucus ... released from private area of women. Sometimes ... it become yellowish. Sometimes, it is white and clear. Yellowish color makes me feel uncomfortable ... it is an infection ... I was young, I saw doctor [in Vietnam] take its sample to exam ... he concluded it is normal white blood ... doctor said that have two kinds ... Vietnamese people call hot. Another one is call physiology, depending on the menstrual period of women, it is secreted.

Another participant described:

As a woman, I am very sensitive in those matters [taking care of white blood] and it is very inconvenient, white blood. Normally, it is dry, but it is wet all the time. I feel uncomfortable ... I must have an exam [CC screening] to see what is the cause and fortune or infortune to find disease .... Generally, women have changes in that private area [cervix], then we need to have an exam [CC screening] immediately ... Having white blood that last long might block ovary tubes that cause infertility or having other dangerous health for uterus; therefore, having exam is better.

\section{Vaginal and/or Perineal Washing to Prevent $C C$ and Infection}

Some participants were aware when to start CC screening. They were also unaware of the recommended frequency of examinations.

\section{Causes and risks of CC.}

The participants expressed several different beliefs about the causes and risks of $\mathrm{CC}$, including an increase in age, heredity, infection with HPV, sexually transmitted disease, multiple sexual partners, smoking, severe urinary tract infection, menorrhagia, high levels of white blood or white discharge, and poor hygiene. Some beliefs about the causes or risks of CC were accurate, many were not.

\section{Private area: To cleanse "women must keep it clean."}

Most VA women discussed the importance of keeping the private area (vaginal and/or perineal areas) clean with thorough washing or soaking to prevent the formation or accumulation of "white blood" or white discharge and also as a means to prevent CC cancer and infection. For example, "Women must keep it [private area] clean. [In Vietnam], there was no discussion much about cancer, but keeping cleanliness ... Keeping it [white blood] long time may cause cancer ... I ... protect myself by doing that way [washing]." Others described the importance of keeping clean through vaginal washing, "... in $\mathrm{Vi}$ etnam, people promoted to use vaginal cleansing water." Another woman shared, "For families with girls, it [vaginal washing] is basic hygiene knowledge that [we] teach girls from early age."

Some VA women discussed using traditional remedies of personal treatment for white blood, rather than going to the hospital, which was referred to as "living in the old times." For example, "[I] soak in coarse salt and boiled water solution." Only a few VA women talked about having a HCP who recommended that if one wanted to cleanse their private areas, then to wash the outside of the vagina only: "Wash outside only. Doctor [in the United States] advises that without having doctor's request, I am not allowed to douche, only wash outside for deodorization or hygiene ... I have opportunities to learn ... I do ... what doctor advises." As described by another:

[When] I was in Vietnam, I had one cervical exam every year. The first time, I was worried its dirtiness and smelliness, then I washed it very clean ... doctor advised that let it [cervix] be natural because my cervix had something to protect ... If I made it very clean, it is easy infected ... I was surprised and said: God! Being clean is not allowed. So, doctor said that it should not be too 
clean or too dry because there is a natural secretion to protect us from bacteria invading.

\section{Limited Cancer Prevention Vaccine Aware- ness and Knowledge}

Most VA women were not familiar with HPV vaccination to prevent cancer and the need for them to promote health:

I heard from my friend, but she just came [immigrated] to the United States, she doesn't understand. They said that schools asked ... because her child is a girl, asking to get $\ldots$ vaccinated for cervical cancer, but I just came [immigrated], I don't understand. Of those who knew about the HPV vaccines, most did not know that the vaccination age range includes young adults: "This vaccine is to prevent cancer. It vaccinates ... children from certain age to certain age, from 12 to 16 years old, I don't remember exactly the ages, but it prevents for teenagers." Only a few women knew that cancer prevention vaccines are for both girls and boys. Whereas, some participants thought that cancer prevention vaccines were only for adolescent girls who are at the age for being sexually active: "I heard that only for girls because having sexual activities is easy to get it .... start reaching to sexual activity ages, get that vaccine to prevent cervical cancer." Some VA women commented that cancer prevention vaccines are not applicable to boys due to their anatomy: "It prevents cervical cancer, but boys don't have cervix."

\section{Decision-Making Influences about Cancer Prevention Vaccines}

Some VA women discussed the various influences on their decision-making or the family decision-making about having their children get vaccinated with cancer prevention vaccines:

I see most Vietnamese women died because of cervical cancer .... At my age, I can't get vaccinated...the vaccine was launched in Vietnam ... I got my daughter vaccinated. She didn't understand about it, I am older, and I see that the vaccine is necessary. Because cancer was found out, people still don't know that is cancer, even doctor ... I know one person, when her cancer metastasized, she didn't know."

A VA woman described her fear and not having the courage to vaccinate her children as the basis for her undecidedness. Talking with a family member and other people may have influenced her fear even though her HCP recommended it:

Doctor is learning to get more understanding [about cancer prevention vaccine], it sounds like it is not safe ... I am afraid; I don't have the courage for my children to vaccinate .... Because I heard ... getting that vaccine [cancer prevention vaccine] for young girls, it will affect their reproduction later that may cause to not have a baby. That is why many people stop and do not allow their children ... I don't know whether having them [my children] getting vaccine or not ... Even my sister, a nurse who is working at a hospital for foreigners in Vietnam, also advises to wait ... her children have not vaccinated yet ... Doctor said, oh, your children should be vaccinated because they are in the range of ages.

In addition to a HCP, a few VA women discussed the role schools play, and how they are expected to be a source of health information including cancer prevention vaccines:

My niece whose age is in the range from 8 to $9 \ldots$ eligible to get vaccinated, but her family did not know anything about this vaccine ... I told her family to ask the school if it has a vaccination program for children. Her father asked doctor, then doctor vaccinated her.

\section{Discussion}

We found that VA women perceive $\mathrm{CC}$ to be a taboo and difficult topic to discuss. This difficulty with the topic also means that they may not inquire about the CC screening, even when they are aware of it. Some VA women experienced intense emotions of fear or shame from having their private area (cervix) examined, which became barriers to $\mathrm{CC}$ screening. The findings suggest that some VA women may be motivated to undergo a CC screening because they know someone who died from the disease. However, having their cervix examined remains an intense fear. Vietnamese women view their body as private (Donnelly, 2006b; Nguyen \& Clark, 2014), and they are hesitant to have a CC screening performed (Donnelly, 2006b). Our findings add to this body of knowledge and to the HBM, which underscores the importance of considering the intensity of a woman's cultural beliefs and emotions when it is recommended to have a cervical exam. HCPs should consider providing culturally sensitive care, whereby VA women are empowered to share if they feel safe in their interactions with HCPs (Doutrich, Arcus, Dekker, Spuck, \& Pollock-Robinson, 2012). The HCPs establishing a safe and comfortable setting for a CC screening dialogue will diminish their fear and negative emotions. Sensitivity to the needs of female HCPs may be helpful with CC screening. Researchers found that CC screening was positively associated with client-HCP gender concordance (Thompson et al., 2014). 
Also, VA women focused on cleanliness maintenance through vaginal and/or perineal washing as a primary prevention for $\mathrm{CC}$. These beliefs highlight the importance of promoting early $\mathrm{CC}$ detection through regular screening, and of clarifying misconceptions or myths. Although some VA women are aware of the signs and symptoms of $\mathrm{CC}$, the current study's findings are of concern and suggest that it may delay $\mathrm{CC}$ screening, leading to delayed $\mathrm{CC}$ diagnosis (i.e., late-stage diagnosis) and treatment in the disease trajectory. The evidence supports the fact that early detection leads to successful CC treatment.

The current study's findings confirm the results from a previous study with VA women about their heavy reliance on personal health regimens including vaginal washing as a means of disease prevention (Gregg et al., 2011). The current study's findings add to the literature through highlighting that "washing" is a cultural tradition that is passed down; for example, from mother to daughter, or shared with friend to friend. Donnelly's study (2006a) with Vietnamese-Canadian immigrant women revealed that women live and practice health care in-between spaces where comparisons and negotiations are being made between the Western and Vietnamese cultures. HCPs, which includes nurses, should take the cultural context of "washing" practices into consideration, to help bridge the health care divide, which could provide opportunities for education and clarification. Shared decision-making would involve a discussion of preferences by both women and their HCPs, and arriving at mutually agreed-upon decisions (Mead et al., 2013).

Of concern was the limited awareness and knowledge about cancer prevention vaccinations, specifically about HPV. Some VA women understood that cancer prevention vaccines were administered to girls, but only a few knew that they were given to boys also and that a series of vaccines was needed to be effective. Some participants believed that cancer prevention vaccines are not safe for their children. Despite HCP recommendations, some participants perceived dangerous side effects posed a considerable barrier to vaccine uptake. The women relied heavily on their informal social networks of family, friends, or community for health knowledge. The current study findings underscore the critical role of HCPs in messaging and promoting cancer prevention vaccine uptake that is culturally and linguistically appropriate for this population. A study with Hispanics, Somalis, and Ethiopians/Eritreans identified insufficient vaccine information available in their native languages as a key barrier to vaccine uptake and completion (Greenfield et al., 2014). VA women are particularly challenged due to suboptimal HCP care (including HPV education and preventive care) and lower English proficiency, which were contribu- tors to communication barriers with HCPs (Yi et al., 2013).

The findings from this study support the need for improvement in community settings' cancer prevention vaccine health education. Studies in Australia with school-based, government-funded vaccine programs have demonstrated that adolescents and their parents have limited understanding about HPV vaccines. Although programs have been successful in increasing HPV vaccine uptake rates that are higher than in the United States, globally adolescents and parents still have difficulty discussing HPV and matters of sexual health (Skinner et al., 2015). The current authors found that VA women value teaching their daughters how to perform daily personal hygiene, suggesting that VA women value familytaught health education. Developing supportive tools for decision-making between parents and adolescents may be beneficial for increasing knowledge of cancer prevention vaccines and screenings (Skinner et al., 2015).

\section{Implications}

We suggest HCPs provide tailored, culturally, and linguistically appropriate risk information when counseling VA women about CC screening and prevention. HCPs have a critical role in being proactive in interventions that create a safe interaction space for communication to promote opportunities to clarify misconceptions and myths.

The current study highlights the family's role in decision-making for CC screening and HPV vaccination, particularly for parents to decide whether or not to have their children vaccinated. HCPs should also consider the role of the family and elicit patients' preferences for family involvement when promoting $\mathrm{CC}$ screening and HPV vaccination. The interests of family members may be an important component of patient-centered care and improved outcomes. Studies are limited evaluating family decision-making among the VA population. The current study provides insights on how families may contribute to the decision-making process with CC screening and HPV vaccines.

A culturally tailored multi-level and multicomponent intervention designed to increase $\mathrm{CC}$ and cancer prevention vaccine knowledge, remove barriers, and connect to vaccination services may improve access to $\mathrm{CC}$ screening and vaccination services. Cancer prevention vaccine uptake may promote positive health outcomes among this population, for example, educational interventions targeting families.

\section{Conclusions}

VA women may experience intense negative emotions of fear or shame of having their private area 
(cervix) examined, creating barriers to CC screening. Although some VA women were aware of the signs and symptoms of $\mathrm{CC}$, the belief in early warning signs to guide health care was a barrier to preventive care. Furthermore, VA women focused on maintaining cleanliness through vaginal and/or perineal washing as their primary prevention for $\mathrm{CC}$. These findings are of concern as VA women may delay $\mathrm{CC}$ screening, possibly resulting in delayed $\mathrm{CC}$ diagnosis and treatment in the disease trajectory. Another concern was the limited awareness and knowledge about the HPV vaccine for cancer prevention. HCPs can create a safe place for addressing preventive measures, ensure privacy in screenings, and provide guidance in making informed decisions.

\section{Acknowledgments}

The authors are grateful to the following for their support in this study: Dr. Frances Lee-Lin, PhD, RN, OCN, CNS, External Research Mentor; Tuyen Tran, MPA-HA, Michelle Tran, Dr. Thuyet Tran, $\mathrm{PhD}, \mathrm{MD}$, Kevin Truong, BS Student, and Katherine Dinh, BS, Community-Participatory Advisory Council Team members of the Vietnamese Women's Health Project community-based participatory research partnership for community outreach, referrals, and debriefing on the study findings; Sophorn Cheang, MBA, BS, Chiao-Yun Hsiao, BS, Thi Luong, Ivy Do, and Linda M. Nguyen, staff at the Immigrant \& Refugee Community Organization Asian Family Center for their community outreach and referrals; and Domenica Searight, BSN, RN, MN student, from Washington State University College of Nursing in Vancouver, for debriefing on the findings. The authors thank Dr. Renee Hoeksel, PhD, RN, ANEF, and Dr. Catherine Van Son, PhD, RN, ANEF, for scientific editing assistance, John Van Son for editing assistance, and the anonymous peer reviewers for assistance.

\section{Declaration of Conflicting Interests}

The author(s) declared no potential conflicts of interest concerning the research, authorship, or publication of this article.

\section{Funding}

Grant funding support from Washington State University Vancouver Research-Mini Grant, Washington State University Vancouver External Mentoring Program, and Washington State University Vancouver New Faculty Research Start-Up Grant, was awarded to Dr. Connie Kim Yen NguyenTruong, PhD, RN, PCCN.

\section{References}

American Cancer Society. (2015). Cancer prevention \& early detection facts \& figures 2015-2016. Retrieved from https://www.cancer.org/research/ cancer-facts-statistics/cancer-prevention-earlydetection.html

Centers for Disease Control and Prevention. (2016). Human Papillomavirus (HPV) vaccination information for clinicians. Retrieved from http:// www.cdc.gov/vaccines/vpd/hpv/hcp/index.html

Champion, V. L., \& Skinner, C. S. (2008). The health belief model. In K. Glanz, B. K. Rimer, \& K. Viswanath (Eds.), Health behavior and health education: Theory, research, and practice (4th ed., pp. 45-65). San Francisco, CA: Jossey-Bass.

Chawla, N., Breen, N., Liu, B., Lee, R., \& Kagawa-Singer, M. (2015). Asian American women in California: A pooled analysis of predictors for breast and cervical cancer screening. American Journal of Public Health, 105, e98-109. https://doi.org/10. 2105/ajph.2014.302250

Donnelly, T. T. (2006a). Living "in-between" - Vietnamese Canadian women's experiences: Implications for health care practice. Health Care for Women International, 27, 695-708. https://doi.org/10. 1080/07399330600817725

Donnelly, T. T. (2006b). The health-care practices of Vietnamese-Canadian women: Cultural influences on breast and cervical cancer-screening. Canadian Journal of Nursing Research, 38, 82-101.

Doutrich, D., Arcus, K., Dekker, L., Spuck, J., \& PollockRobinson, C. (2012). Cultural safety in New Zealand and the United States: Looking at a way forward together. Journal of Transcultural Nursing, 23, 143-150. https://doi.org/10.1177/ 104365 9611433873

Gomez, S. L., Noone, A.-M., Lichtensztajn, D. Y., Scoppa, S., Gibson, J. T., Liu, L., ... Miller, B. A. (2013). Cancer incidence trends among Asian American populations in the United States, 1990-2008. Journal of the National Cancer Institute, 105, 1096-1110. https:// doi.org/10.1093/jnci/djt157

Greenfield, L. S., Page, L. C., Kay, M., Li-Vollmer, M., Breuner, C. C., \& Duchin, J. S. (2015). Strategies for increasing adolescent immunizations in diverse ethnic communities. Journal of Adolescent Health, 56, S47-S53. https://doi.org/10.1016/j. jadohealth.2014.10.274

Gregg, J., Nguyen-Truong, C. K. Y., Wang, P., \& Kobus, A. (2011). Prioritizing prevention: Culture, context, and cervical cancer screening among Vietnamese American women. Journal of Immigrant and Minority Health, 13, 1084-1089. https:// doi.org/10.1007/s10903-011-9493-2

HealthyPeople.gov. (2017). C-15: Increase the proportion of women who receive a cervical cancer screening based on the most recent guidelines. Retrieved from https://www.healthypeople.gov/ node/4053/data_details

Hennink, M. M., Kaiser, B. N., \& Marconi, V. C. (2017). Code saturation versus meaning saturation: How many interviews are enough? Qualitative Health 
Research, 27, 591-608. https://doi.org/10.1177/ 1049732316665344

Ho, V., Yamal, J. M., Atkinson, E. N., Basen-Engquist, K., Tortolero-Luna, G., \& Follen, M. (2005). Predictors of breast and cervical screening in Vietnamese women in Harris County, Houston, Texas. Cancer Nursing, 28, 119-129.

Hsieh, H. F., \& Shannon, S. E. (2005). Three approaches to qualitative content analysis. Qualitative Health Research, 15, 1277-1288. https://doi.org/10.1177/ 1049732305276687

Immigrant \& Refugee Community Organization. (2017). Mission \& history. Retrieved from https://irco. org/who-we-are/mission-history.html

Israel, B. A., Eng, E., Schulz, A. J., \& Parker, E. A. (2012). Introduction to methods in community-based participatory research for health. In B. A. Israel, E. Eng, A. J. Schulz, \& E. A. Parker (Eds.), Methods in community based participatory research for health (pp. 3-37). San Francisco: Jossey-Bass.

Lee, H. Y., Kwon, M., Vang, S., DeWolfe, J., Kim, N. K., Lee, D. K., \& Yeung, M. (2015). Disparities in human papillomavirus vaccine literacy and vaccine completion among Asian American Pacific Islander undergraduates: Implications for cancer health equity. Journal of American College Health, 63, 316-323. https://doi.org/10.1080/ 07448481.2015 .1031237

Lee-Lin, F., Menon, U., Nail, L., \& Lutz, K. F. (2012). Findings from focus groups indicating what Chinese American immigrant women think about breast cancer and breast cancer screening. Journal of Obstetric, Gynecologic \& Neonatal Nursing, 41, 627-637. https://doi.org/10.1111/j.15526909.2012.01348.x

Lee-Lin, F., Nguyen, T., Pedhiwala, N., Dieckmann, N., \& Menon, U. (2015). A breast health educational program for Chinese American women: 3- to 12month postintervention effect. American Journal of Health Promotion, 29, 173-181. https://doi.org /10.4278/ajhp.130228-quan-91

Lincoln, Y. S., \& Guba, E. G. (1985). Establishing trustworthiness. In Naturalistic inquiry (pp. 289-331). Beverly Hills, CA: Sage.

Mead, E. L., Doorenbos, A. Z., Javid, S. H., Haozous, E. A., Alvord, L. A., Flum, D. R., \& Morris, A. M. (2013). Shared decision-making for cancer care among racial and ethnic minorities: A systematic review. American Journal of Public Health, 103(12), e15-29. https://doi.org/10.2105/aiph. $\underline{2013.301631}$

Miller, B. A., Chu, K. C., Hankey, B. F., \& Ries, L. A. G. (2008). Cancer incidence and mortality patterns among specific Asian and Pacific Islander populations in the U.S. Cancer Causes \& Control, 19, 227-256. https://doi.org/10.1007/s10552-007-90 $\underline{88-3}$

Morgan, D. L., \& Bottorff, J. L. (2010). Advancing our craft: Focus group methods and practice. Qualitative Health Research, 20, 579-581. https://doi. org/10.1177/1049732310364625

Nguyen, A. B., \& Clark, T. T. (2014). The role of acculturation and collectivism in cancer screening for
Vietnamese American women. Health Care for Women International, 35, 1162-1180. https://doi. org/10.1080/07399332.2013.863317

Nguyen-Truong, C. K. Y., Lee-Lin, F., Leo, M. C., GedalyDuff, V., Nail, L. M., Wang, P., \& Tran, T. (2012). A community based participatory research approach to understanding Pap testing adherence among Vietnamese American immigrants. Journal of Obstetric, Gynecologic \& Neonatal Nursing, 41, E26-40. https://doi.org/10. 1111/j.1552-6909.2012.01414.x

Nguyen-Truong, C. K. Y., Leo, M. C., Lee-Lin, F., GedalyDuff, V., Nail, L. M., Gregg, J., ... Tran, T. (2015). Adaptation and testing of instruments to measure cervical cancer screening factors among Vietnamese immigrant women. Journal of Transcultural Nursing, 26, 244-253. https://doi.org/ $10.1177 / 1043659614524245$

Nguyen-Truong, C. K. Y., Tang, J., \& Hsiao, C-Y. (2017). Community interactive research workshop series: Community members engaged as team teachers to conduct research. Progress in Community Health Partnerships: Research, Education, and Action, 11, 215-221. https://doi.org/10.1353/cpr. 2017.0026

Skinner, S. R., Davies, C., Cooper, S., Stoney, T., Marshall, H., Jones, J., ... McGeechan, K. (2015). HPV.edu study protocol: A cluster randomised controlled evaluation of education, decisional support and logistical strategies in school-based human papillomavirus (HPV) vaccination of adolescents. BMC Public Health, 15(896). https://doi.org/10. 1186/s12889-015-2168-5

Solanki, P. A., Ko, N. Y., Qato, D. M., \& Calip, G. S. (2016). Risk of cancer-specific, cardiovascular, and all-cause mortality among Asian and Pacific Islander breast cancer survivors in the United States, 1991-2011. SpringerPlus, 5(82). https:// doi.org/10.1186/s40064-016-1726-3

Strong, C., Lee, S., Tanaka, M., \& Juon, H-S. (2012). Ethnic differences in prevalence and barriers of HBV screening and vaccination. Journal of Community Health, 37, 1071-1080. https://doi.org/10.1007/ s10900-012-9541-4

Thompson, C. A., Gomez, S. L., Chan, A., Chan, J. K., McClellan, S. R., Chung, S., ... Palaniappan, L. P. (2014). Patient and provider characteristics associated with colorectal, breast, and cervical cancer screening among Asian Americans. Cancer Epidemiology, Biomarkers \& Prevention, 23, 2208-2217. https://doi.org/10.1158/1055-9965. epi-14-0487

Wiggins, N. (2011). Popular education for health promotion and community empowerment: A review of the literature. Health Promotion International, 27, 356-371. https://doi.org/10.1093/heapro/dar $\underline{046}$

Yi, J. K., Anderson, K. O., Le, Y-C., Escobar-Chaves, S. L., \& Reyes-Gibby, C. C. (2013). English proficiency, knowledge, and receipt of HPV vaccine in Vietnamese-American women. Journal of Community Health, 38, 805-811. https://doi.org/ $\underline{10.1007 / \mathrm{s} 10900-013-9680-2}$ 